\title{
Coherent Population Trapping Resonances in Cs Atomic Vapor Layers of Micrometric Thickness
}

\author{
A. Krasteva, D. Slavov, and S. Cartaleva \\ Institute of Electronics, Bulgarian Academy of Sciences, 72 Tsarigradsko Chaussee Boulevard, 1784 Sofia, Bulgaria \\ Correspondence should be addressed to S. Cartaleva, stefka-c@ie.bas.bg
}

Received 29 April 2011; Revised 12 June 2011; Accepted 19 June 2011

Academic Editor: Adrian Podoleanu

Copyright () 2011 A. Krasteva et al. This is an open access article distributed under the Creative Commons Attribution License, which permits unrestricted use, distribution, and reproduction in any medium, provided the original work is properly cited.

We report on a novel behavior of the electromagnetically induced absorption (EIA) resonance observed on the $D_{2}$ line of Cs for atoms confined in cells with micrometric thickness. With the enhancement of light intensity, the EIA resonance amplitude suffers from fast reduction, and even at very low intensity $\left(W<1 \mathrm{~mW} / \mathrm{cm}^{2}\right)$, resonance sign reversal takes place and electromagnetically induced transparency (EIT) resonance is observed. Similar EIA resonance transformation to EIT one is not observed in conventional $\mathrm{cm}$-size cells. A theoretical model is proposed to analyze the physical processes behind the EIA resonance sign reversal with light intensity. The model involves elastic interactions between Cs atoms as well as elastic interaction of atom micrometric-cell windows, both resulting in depolarization of excited state which can lead to the new observations. The effect of excited state depolarization is confirmed also by the fluorescence (absorption) spectra measurement in micrometric cells with different thicknesses.

\section{Introduction}

Coherent population trapping (CPT) resonances prepared in the Hanle configuration have been widely investigated for Cs atoms confined in optical cells with centimeter dimensions. Alkali atoms, imposed on variable magnetic field $B$, are irradiated by monochromatic laser field in a way that different light polarization components couple Zeeman sublevels of one hyperfine ground state to a common excited state. In this way, the coherence is introduced between the ground-state magnetic sublevels at $B=0$. As has been shown in $[1,2]$, in absence of depolarizing collisions of the excited state, depending on the degeneracy ratio of the two levels involved in the optical transition, subnatural width resonance of both electromagnetically induced transparency (EIT or dark) resonance and electromagnetically induced absorption (EIA or bright) resonance can be observed. The EIT is realized when the condition $F_{g} \rightarrow F_{e}=\left(F_{g}-1\right)$, $F_{g}$ is met, while EIA is observed for $F_{g} \rightarrow F_{e}=F_{g}+$ 1 kind of transitions. Here, $F_{g}$ and $F_{e}$ are the hyperfine quantum numbers of the ground and excited hyperfine levels, respectively.
The relevant Cs energy levels involved are illustrated in Figure 1. The two groups of transitions, starting from $F_{g}=3$ and $F_{g}=4$ levels, are denoted. In case of optical cell with thickness $L$ of several centimeters and transversal dimensions of few centimeters (called further conventional cell), the open hyperfine transitions are depleted due to the hyperfine optical pumping to the ground hyperfine level noninteracting with the laser light and do not play significant role in the formation of the CPT resonance. At the same time, the closed transition that does not suffer hyperfine optical pumping mainly determines the sign of the CPT resonance. Thus, dark resonance has been observed at the absorption (fluorescence) line starting from the $F_{g}=3$ level and bright at that starting from the $F_{g}=4$ level [2].

In the recently developed extremely thin cell (ETC) $[3,4]$, with transversal dimensions of few centimeters but with thickness $L=\lambda$ ( $\lambda$-the light wavelength), also dark resonance is observed for the $F_{g}=3$ set of transitions [5]. However, in such cell, the bright resonance sign reversal has been reported [5], for the $F_{g} \rightarrow F_{e}=F_{g}+1$ transitions. A theoretical model has been proposed that involves the elastic interaction of atoms confined in the ETC with its walls 


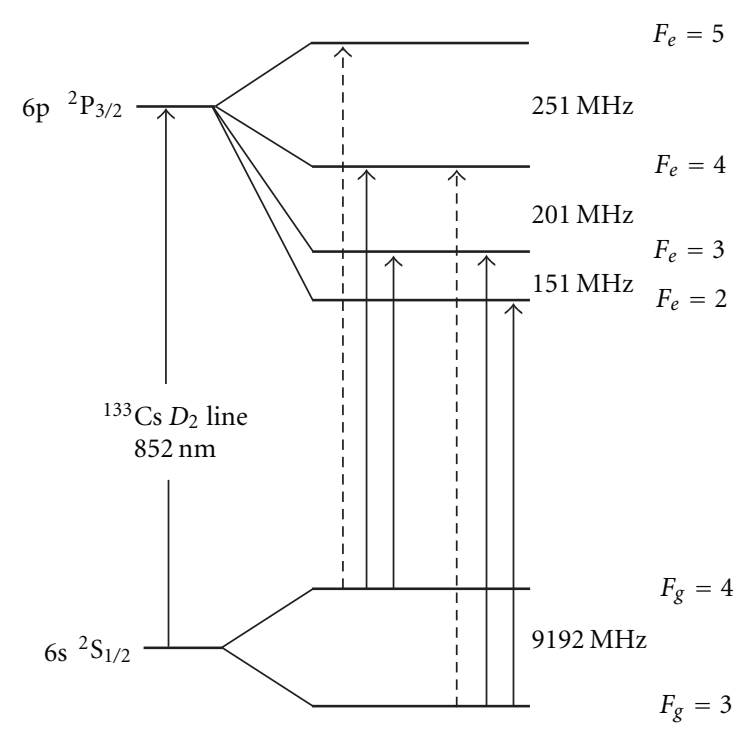

Figure 1: Energy-level diagram for $D_{2}$ line of Cs. The $F_{g} \rightarrow F_{e} \leq F_{g}$ transitions (solid line) are distinguished from the $F_{g} \rightarrow F_{e}>F_{g}$ transitions (dashed line).

resulting in depolarization of the excited state, which leads to the EIA resonance sign reversal.

Here, we report on the CPT resonance study related to the $D_{2}$ line of Cs vapor confined in a cell with micrometric thickness $(L=700 \mu \mathrm{m})$ and cell window diameter of about $1 \mathrm{~cm}$. For the $F_{g} \rightarrow F_{e}=F_{g}+1$ kind of transitions, the EIA resonance transformation into EIT one is observed experimentally with the light intensity increasing. To analyze the resonance sign reversal, a theoretical modeling is performed describing the atomic population distribution among the magnetic sublevels of hyperfine levels, forming the optical transition.

The absorption and fluorescence spectra from micrometric thickness cells with different levels of dimensional anisotropy (i.e., $L=5 \mu \mathrm{m}$ and $L=700 \mu \mathrm{m}$ ) are also analyzed. It is shown that these spectra present as well experimental evidence for the influence of the cell windows to the excited state depolarization. The results for the micrometric thickness cells are compared to those obtained in conventional cell.

The micrometric-thickness-cell study is of significant importance for miniaturization of practical photonics sensors based on the CPT phenomenon. Such sensors are of recent interest for precise frequency standards, atomic clocks, and optical magnetometers [6].

\section{Principal Experimental Results: Coherent Resonance Behavior}

2.1. Description of Experimental Setup. Our experimental setup, presented in Figure 2, is similar to those used in $[2,5]$. The beam of a narrow-band diode laser, operating in single-frequency mode at $\lambda=852 \mathrm{~nm}$, is directed to the cell in orthogonal to the cell window direction. The light polarization is linear. Three different kinds of cells are used separately: (i) conventional cell with $L=2.5 \mathrm{~cm}$, (ii) thin cell with $L=700 \mu \mathrm{m}$, and (iii) ETC with $L \approx 5 \mu \mathrm{m}$. All the cells are evacuated, and optical glass cells without buffer gas added and are filled by Cs vapor from a small Cs container situated in a side arm connected to the cell.

Cs atomic vapor transmission or absorption is measured versus a magnetic field $B$ applied in a collinear to the laser beam direction. The $B$ field is produced by two coils arranged in the Helmholtz configuration. The magnetic field amplitude is varied around $B=0$. The experiment is performed without stray magnetic field shielding of the optical cell.

First, the CPT resonance profile dependence on light intensity is measured for the $L=700 \mu \mathrm{m}$ cell, and then its behavior is compared to that of the conventional cell, where the CPT resonance profile is registered under the same experimental conditions, including the stray magnetic field. In an additional experiment, the transmission and the fluorescence spectra of the $D_{2}$ line of Cs are recorded, at $B \approx 0$, for $L=700 \mu \mathrm{m}$ and $L=6 \lambda$ cells.

The used in our experiment optical cell represents, in fact, a multipurpose cell having three sectors with different thicknesses (see Figure 2), that is, with the same Cs-vapor pressure in each sector. The construction of such a multipurpose cell is proposed in [7]. It is built by two YAG plates separated from each other at about $5 \mu \mathrm{m}$, thus forming the ETC with $L=6 \lambda$. Two apertures are made in one of the plates allowing the formation of two additional sectors with different thicknesses $L=700 \mu \mathrm{m}$ and $L=2 \mathrm{~mm}$, respectively. In the presented work, the $L=700 \mu \mathrm{m}$ and $L=6 \lambda$ regions of the cell are investigated.

2.2. Laser Frequency Tuning to the $F_{g}=3 \rightarrow F_{e}=2,3,4$ Set of Transitions. Here, we present the CPT resonance observed in the Hanle configuration and $L=700 \mu \mathrm{m}$ cell, for the $F_{g}=$ 3 set of transitions, that is, the group of hyperfine optical transitions starting from the $F_{g}=3$ level. Similar to previous measurements for both conventional cell [2] and ETC [5], for the $L=700 \mu \mathrm{m}$ cell also dark resonance is observed at the $F_{g}=3$ set of transitions (Figure 3 ). In our experiment, the CPT resonance width in the conventional cell is more than 30 times less than that observed in the ETC with $L=\lambda$ [5]. The explanation of the strong resonance broadening in the ETC is related to the ground-state hyperfine level broadening due to the significantly increased rate of inelastic atomic collisions with the cell walls.

Due to that result [5], we expected some CPT resonance broadening also for the case of the thin cell. However, our experiments have shown that at the $F_{g}=3$ set of transitions, the resonance width and contrast observed in the $L=$ $700 \mu \mathrm{m}$ cell is similar to that of the resonance prepared in the conventional cell under the same experimental conditions. Hence, the reduction of cell thickness down to several hundreds of microns does not compromise the resonance parameters. Moreover, the $L=700 \mu \mathrm{m}$ cell is much less sensitive to magnetic field gradients, which could be advantageous for application in optical magnetometers with high spatial resolution [6]. For both conventional and 


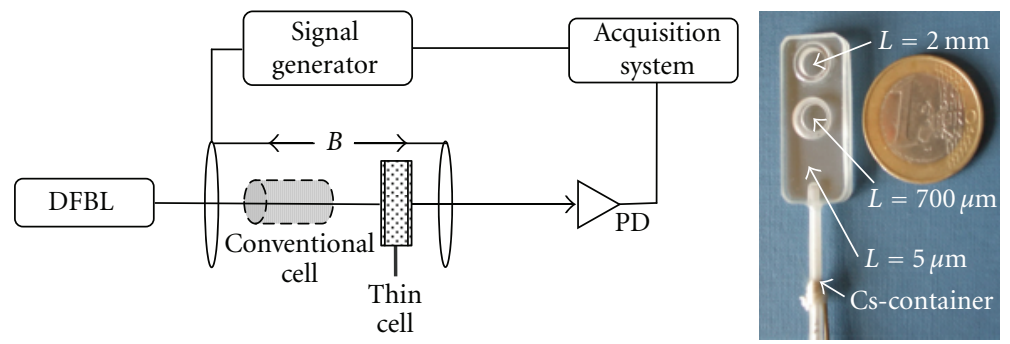

FIGURE 2: Principal scheme of the experimental setup (left) (DFBL: distributed feedback laser, PD: photodiode, $B$ : magnetic field directions) and photo of the optical cell used in the experiment (right).

$L=700 \mu \mathrm{m}$ cells, the CPT resonance broadening and its contrast enhancement with laser power density $W$ take place (Figure 3).

2.3. Laser Frequency Tuning to the $F_{g}=4 \rightarrow F_{e}=3,4,5$ Transitions. For the $L=700 \mu \mathrm{m}$ cell and the $F_{g}=4$ set of transitions, the situation is more complicated. Here at low light intensity $\left(W \sim 0.08 \mathrm{~mW} / \mathrm{cm}^{2}\right)$, a narrow EIA resonance is observed, which is superimposed on the bottom of an EIT resonance. With the enhancement of laser intensity, the bright resonance amplitude suffers fast reduction and even at still very low intensity $\left(W<1 \mathrm{~mW} / \mathrm{cm}^{2}\right)$, the resonance sign reversal takes place, that is, the EIA resonance transforms into an EIT one. Figure 4(a) illustrates the observed phenomenon presenting the resonance profiles in absolute absorption units. For better comparison, a zoom of experimental curves is shown in Figure 4(b).

In case of conventional cells, the reduction of the bright resonance amplitude with light intensity has been predicted by Renzoni at al. [8] theoretically. There, the modeling has been performed for pure homogeneous broadening of the optical transitions. The bright resonance vanishing with intensity was attributed to the optical transition saturation. Later on, an experiment performed with conventional cell containing pure Cs (where Doppler broadening of hyperfine transition is much larger than the homogeneous one) has shown that the EIA resonance is observed up to power density larger than $200 \mathrm{~mW} / \mathrm{cm}^{2}$ [2].

To confirm previous experimental results in more definite way, the CPT resonance profile was measured on the $F_{g}=4$ set of transitions for the conventional cell, under the same experimental conditions as for the $L=700 \mu \mathrm{m}$ cell. As can be seen from Figure 5, no bright resonance sign reversal with the light intensity occurs for the conventional cell. Our experiments have shown no transformation of the EIA resonance to EIT one up to light intensities about two orders of magnitude higher than the lowest intensity shown in Figure 5.

2.4. Discussion of the Experimental Results. As mentioned above for the case of $L=\lambda$ cell thickness, only EIT resonance has been observed on the $F_{g}=4 \rightarrow F_{e}=5$ transition [5]. In that paper, for the explanation of the EIA resonance transformation into an EIT one, the elastic interaction processes of atoms with ETC walls (i.e., windows) were incorporated. The assumed elastic collisions of Cs atoms with the cell windows do not affect the ground state of alkalimetal atoms and do not reorient the electronic and nuclear spins of the atom. However, this kind of collision does affect the excited-state Zeeman coherences and populations, as well as the optical coherences. The involvement of elastic collisions of this type results in depolarization of the Cs excited state that has been polarized by the exciting radiation. This depolarization leads to the accumulation of atomic population in ground-state Zeeman sublevels with the lowest probability of excitation. Note that it is opposite to the situation in the conventional cell, where atoms accumulate on the Zeeman sublevel possessing the largest probability of excitation, which results in the EIA resonance observation $[2,8]$.

Hence, in relation to the CPT resonance preparation on the $F_{g} \rightarrow F_{e}=F_{g}+1$ kind of transitions, the obtained experimental results allow us to consider, from one hand, the $L=700 \mu \mathrm{m}$ cell as one involving the properties of both conventional cell and ETC. From the other hand, however, no transformation of the EIA to EIT resonance with laser light intensity has been observed in the previous experimental investigations.

We can summarize the possible factors responsible for the different behavior of the CPT resonance in the three types of cells as it follows:

(i) different saturation processes of the optical transitions in the conventional cell, $L=700 \mu \mathrm{m}$ cell, and ETC. While the conventional cell can be characterized by the steady state equations; for the ETC the dynamic of absorption-emission processes could result in a lower contribution of the Doppler broadening and, hence, higher rate of optical transition saturation;

(ii) the second reason is related to the influence of cell windows to the polarization of the excited atomic state. The assumption of such influence still needs unambiguous experimental confirmation;

(iii) the third possible reason is the elastic interaction between Cs atoms. The strength of this factor is strongly dependent on the cell temperature, which determines the Cs vapor pressure and atomic density. At the atomic source temperatures close to the room temperature, the number of Cs atoms strongly decreases, making this factor negligible. 


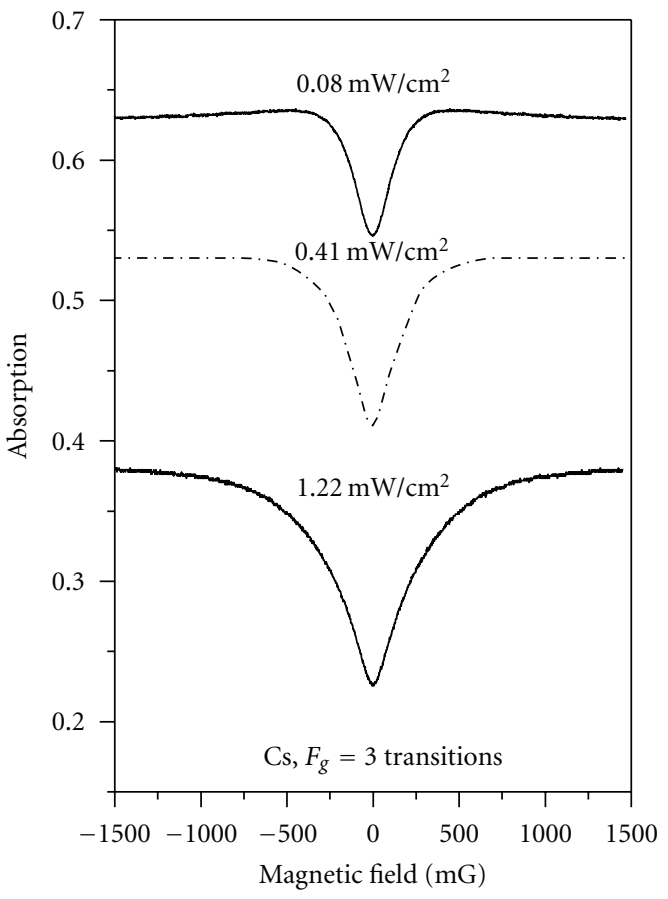

(a)

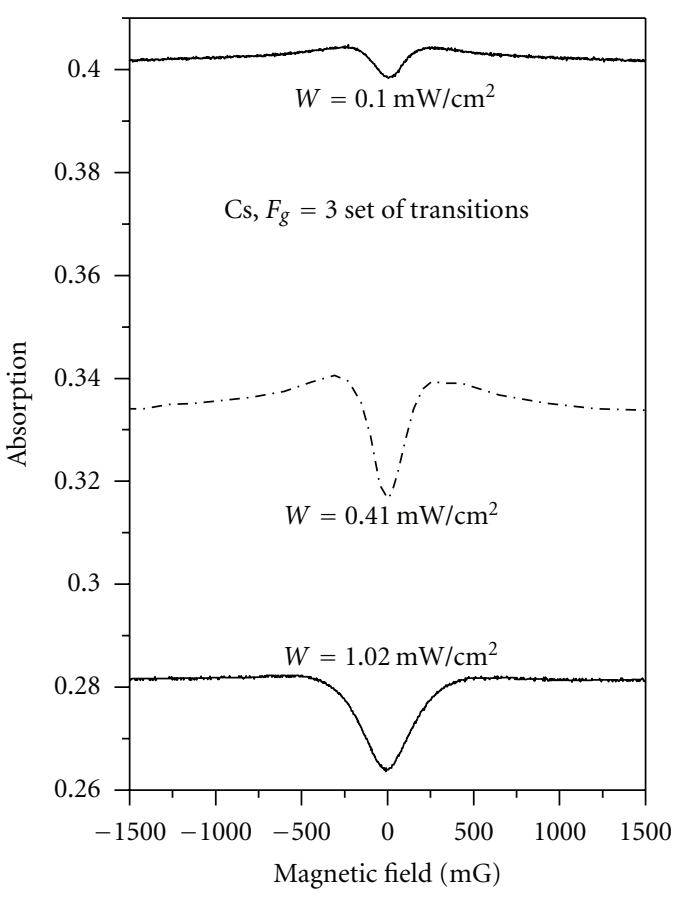

(b)

FIGURE 3: CPT dark resonance profile with laser power density enhancement observed at the $F_{g}=3$ set of transitions: (a) conventional cell with $L=2.5 \mathrm{~cm}$ and atomic source temperature $T=22^{\circ} \mathrm{C}$; (b) thin cell with $L=700 \mu \mathrm{m}$ at $T=34^{\circ} \mathrm{C}$. In both cells, no resonance sign change is observed.

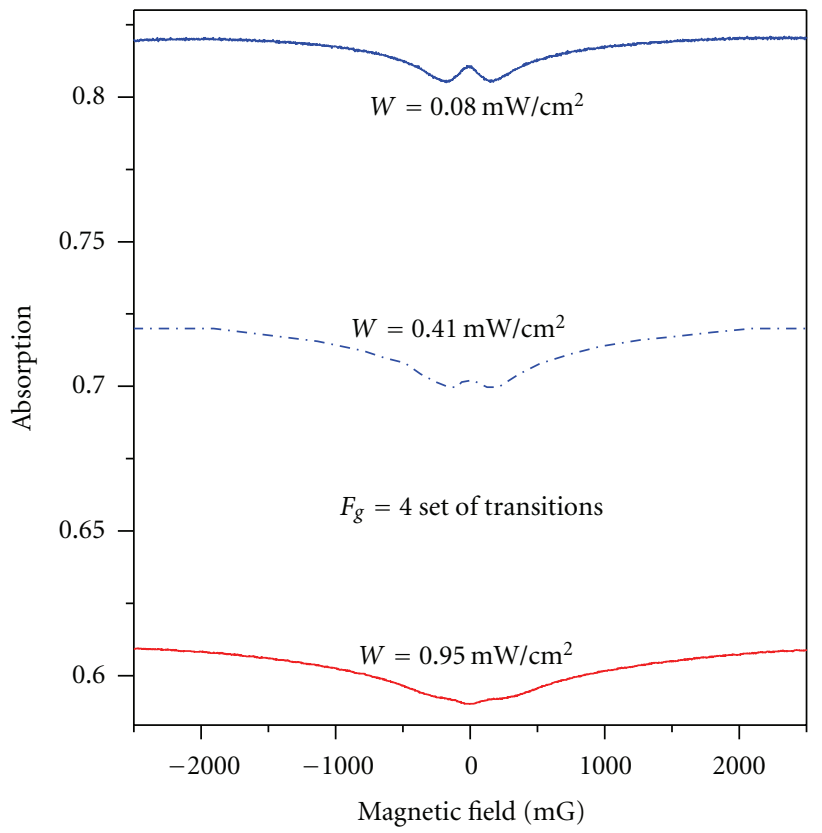

(a)

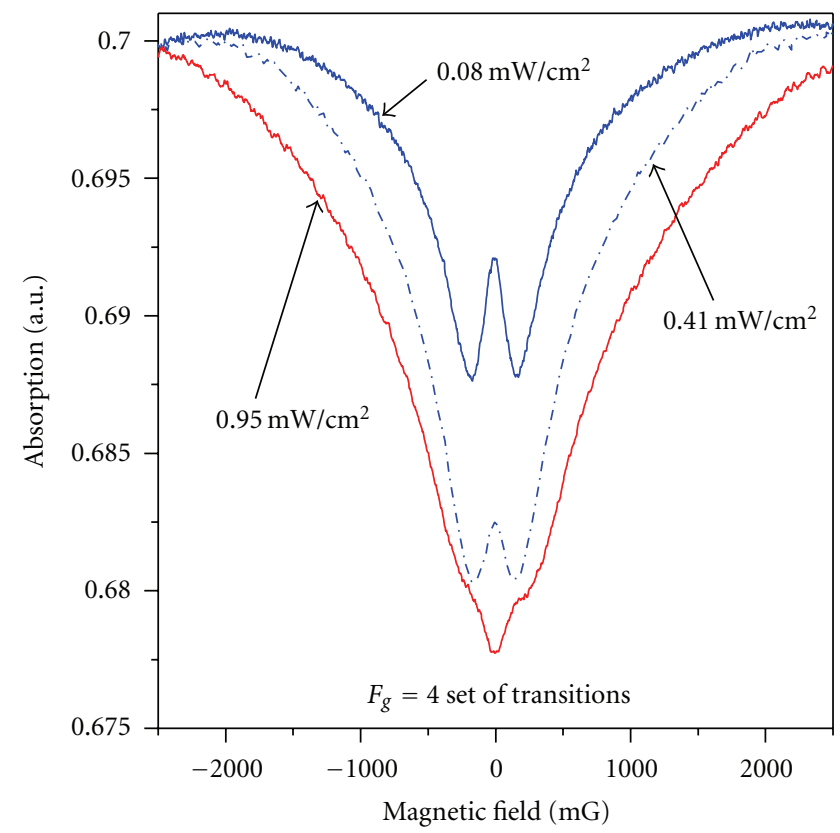

(b)

FIGURE 4: CPT bright resonance sign reversal with laser power density observed on the $D_{2}$ line of Cs at the $F_{g}=4$ set of hyperfine transitions. Thin cell with $L=700 \mu \mathrm{m}$ and $T=34^{\circ} \mathrm{C}$; CPT resonance profile (a) presented in absolute absorption scale; (b) zoomed-in arbitrary absorption units. 


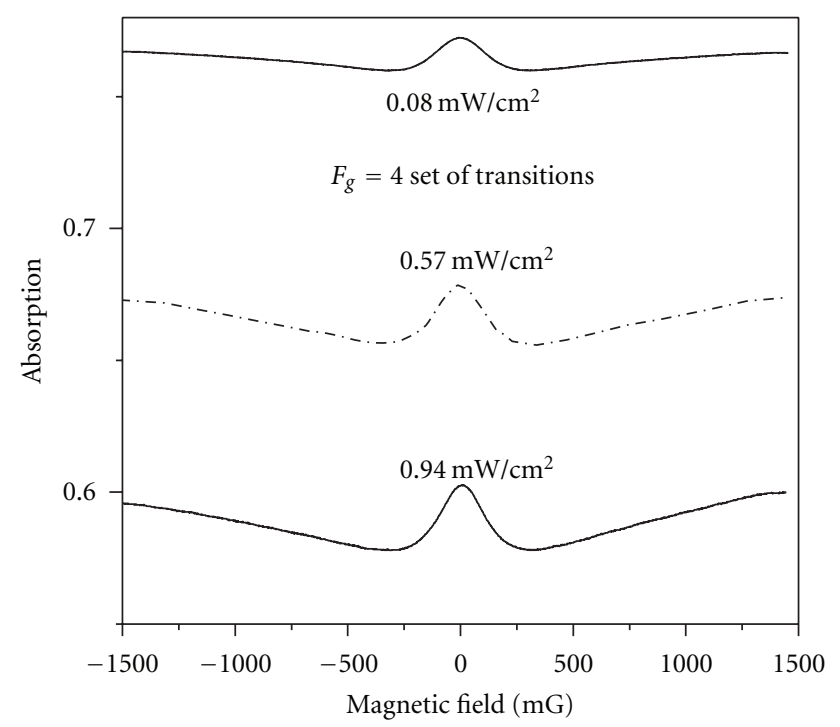

Figure 5: CPT bright resonance profile with light intensity, at the $F_{g}=4$ set of transitions. The conventional cell is at room temperature $\left(T=22^{\circ} \mathrm{C}\right)$.

Theoretical modeling is further performed in order to analyze the physical processes behind the EIA resonance transformation into EIT one with light intensity, which was observed experimentally for the $L=700 \mu \mathrm{m}$ cell.

\section{Theoretical Model}

3.1. Description of the Model. In order to illustrate the process of EIA resonance formation for the system in steady state, we calculate first the atomic population density on each magnetic sublevel of the ground and excited levels of the $F_{g}=$ $4 \rightarrow F_{e}=5$ transition. We formulate and solve the system of rate equations describing the evolution of the population of the magnetic sublevels as a result of the spontaneous and stimulated transitions from the ground and excited levels. Linear polarization of the laser beam is considered with quantization axis along the polarization vector. The system of 18 equations is presented as follows:

$$
\begin{aligned}
\frac{\partial N\left\{m_{g_{i}}\right\}}{\partial t}= & A_{i} s\left(N\left\{m_{e_{i}}\right\}-N\left\{m_{g_{i}}\right\}\right)+A_{i} N\left\{m_{e_{i}}\right\} \\
& +C_{i} N\left\{m_{e_{i-1}}\right\}+B_{i} N\left\{m_{e_{i+1}}\right\}, \\
\frac{\partial N\left\{m_{e_{i}}\right\}}{\partial t}= & A_{i} s\left(N\left\{m_{g_{i}}\right\}-N\left\{m_{e_{i}}\right\}\right)-A_{i} N\left\{m_{e_{i}}\right\} \\
& -C_{i+1} N\left\{m_{e_{i}}\right\}-B_{i-1} N\left\{m_{e_{i}}\right\} .
\end{aligned}
$$

Here $N\left\{m_{g_{i}}\right\}$ and $N\left\{m_{e_{i}}\right\}$ are the populations of the magnetic sublevels of the ground and of the excited levels of the ${ }^{133} \mathrm{Cs} \mathrm{D}_{2}$ line, respectively. The coefficients $A, B$, and $C$ are the square of the dipole matrix element respectively for: $\pi$ transitions $\left(m_{F^{\prime}}=m_{F}\right), \sigma^{+}$-transitions $\left(m_{F^{\prime}}=m_{F+1}\right)$, and $\sigma^{-}$-transitions $\left(m_{F^{\prime}}=m_{F-1}\right)$ [9]. With $i=0, \pm 1, \pm 2, \pm 3$, \pm 4 we denote the number of the corresponding Zeeman sublevel. The initial conditions of the rate equation system are the following.

(i) At the initial moment $t=0$, all the atoms are equally distributed between the magnetic sublevels of the ground level.

(ii) The system is closed, that is, the sum of the population of the ground and excited states $N_{\text {tot }}=1$.

(iii) The system is in steady state, that is, the population of a given Zeeman sublevel does not change in time.

(iv) The calculations are performed with the saturation coefficient $s=I / I_{s}=0.1$.

3.2. Atomic Population Distribution without Depolarization of the Excited State. Based on (1), the population of magnetic sublevels of the $F_{g}=4 \rightarrow F_{e}=5$ transition is calculated. The calculations show (Figure 6 ) that the $F_{g}=4$ level population is accumulated mainly on the magnetic sublevels with $i=$ $0, \pm 1$. Note that these sublevels are the most strongly coupled to Zeeman sublevels of the $F_{e}=5$ level. The transitions from these sublevels have the highest Clebsch-Gordon coefficient (see Figure 7) and, hence, higher transition probability [9]. This fact directly leads to an increase of the fluorescence (absorption) signal at $B=0$. If the magnetic field, which is orthogonal to the atomic alignment, is applied and increased, the atomic population on the $F_{g}=4$ level will evolve to the magnetic sublevels with lower probability of excitation; thus, resulting in lower fluorescence (absorption). In this way, an intuitive explanation of the formation of EIA resonance is presented.

3.3. Calculation of the Excited State Depolarization. As pointed out above for the ETC (with $L=\lambda$ ) and $F_{g} \rightarrow F_{e}=$ $F_{g}+1$ transitions, the EIA resonance transforms into EIT resonance. This effect is attributed to the depolarization of the excited state, as a result of the elastic interactions between Cs atoms and the windows of the ETC [5]. Similarly, elastic interaction can take place between Cs atoms as well. The result of these elastic interactions is a mixing of the excited magnetic sublevel populations. Such mixing leads to atomic accumulation on the magnetic sublevels of the ground level, which are with the lowest probability of excitation. Thus at $B=0$, the atomic absorption is minimal, while it is enhanced with the magnetic field application. Hence, the depolarization of the excited state manifests itself as transformation of the EIA resonance to EIT one.

To estimate the collision rate between Cs atoms, and hence the excited state depolarization, we need first to know the mean free path of the Cs atoms. Using the Cs vapor pressure $P(T)$ [9] and Cs vapor density $\rho(T)$; having the relation $\rho(T)=P(T) / k_{B} T$, we calculate the mean free path between the collisions of two Cs atoms, using the relation $\lambda=\bar{v} / r_{\text {coll }}[10]$, with $\bar{\nu}$-the most probable velocity of the Cs atoms and $r_{\text {coll }}$ : the frequency of the elastic collisions, $k_{B}$ : the Boltzmann constant, and $T$ : the atomic source temperature. Finally, we will estimate the number of elastic collisions and we will analyze the resulting depolarization.

The following constants are used during the calculation: $k_{B}=13.8 * 10^{-24} \mathrm{~J} / \mathrm{K} ; M=2.207 * 10^{-25} \mathrm{~kg}$-the Cs-atom 


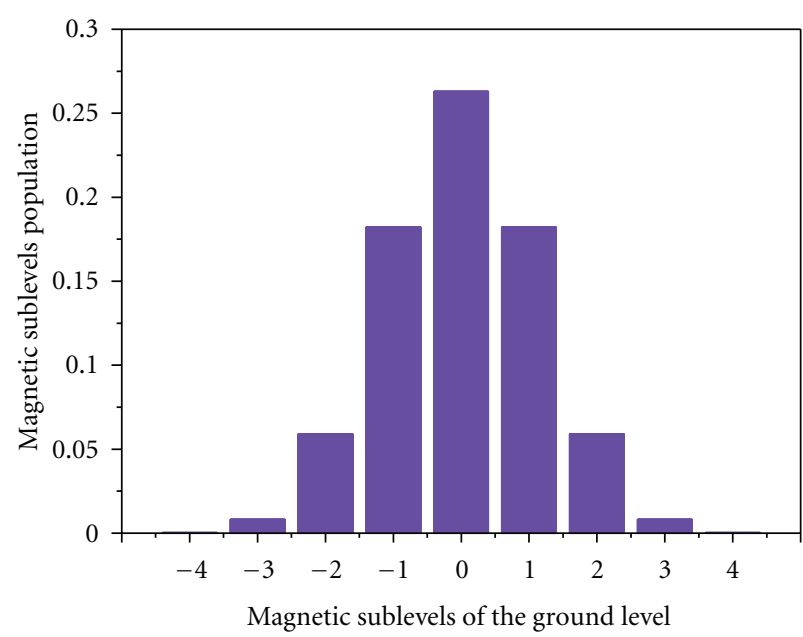

(a)

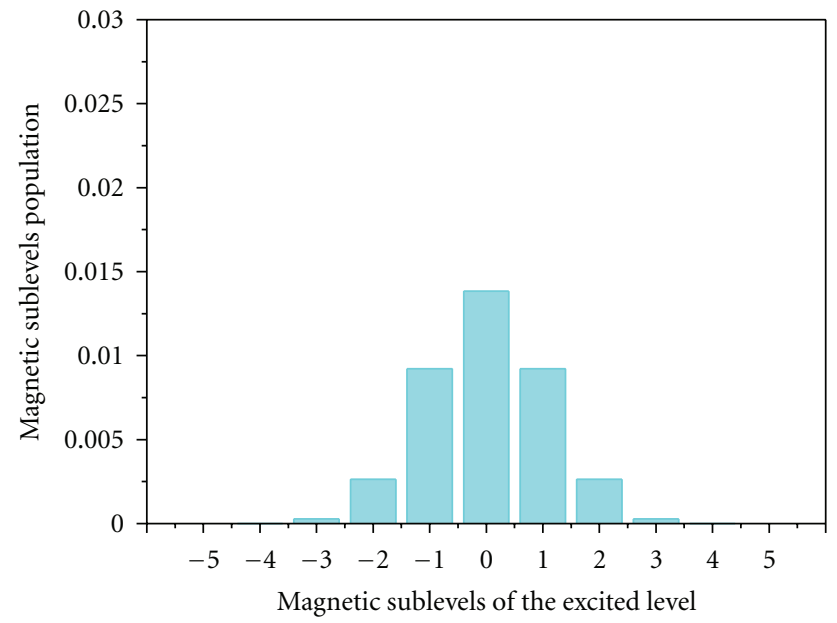

(b)

FIGURE 6: Illustration of the population distribution between the Zeeman sublevels of the ground level $F_{g}=4$ (a) and of the excited level $F_{e}=5$ (b) for the $F_{g}=4 \rightarrow F_{e}=5$ transition, without depolarization factors influencing the excited level. The ground-level population is mainly accumulated on the sublevels $m_{F}=0, \pm 1$, having the highest excitation probabilities (Clebsch-Gordon coefficients).

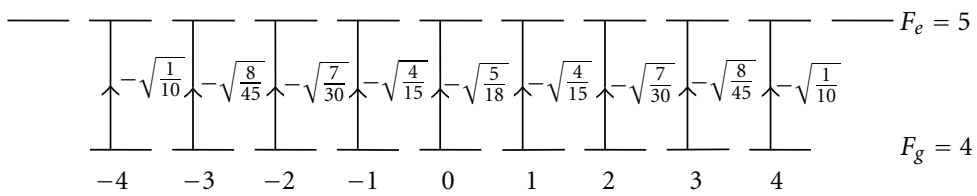

Figure 7: Clebsch-Gordon coefficients describing the transition probabilities between Zeeman sublevels of the ground and excited levels of the $F_{g}=4 \rightarrow F_{e}=5$ transition for linear polarization of the laser light [8].

mass; $\sigma_{k}=1 * 10^{-15} \mathrm{~m}^{2}$ - Cs-atoms collision cross section; $d=1 * 10^{-2} \mathrm{~m}$-thin-cell window diameter.

Table 1 presents the calculated parameters for two different atomic source temperatures.

At the atomic source temperature $T_{1}=120^{\circ} \mathrm{C}$, from Table 1 we can conclude that the number of the elastic collisions is essential and can also provide the mixing of the excited level magnetic sublevel population, leading to the excited level depolarization. Figure 8 illustrates the process of the population mixing.

In order to estimate the excited state depolarization, we formulate and solve a modified system of differential equations involving atomic population mixing between the magnetic sublevels of the excited level. The system of equations is the following:

$$
\begin{aligned}
\frac{\partial N\left\{m_{g_{i}}\right\}}{\partial t}= & A_{i} s\left(N\left\{m_{e_{i}}\right\}-N\left\{m_{g_{i}}\right\}\right)+A_{i} N\left\{m_{e_{i}}\right\} \\
& +C_{i} N\left\{m_{e_{i-1}}\right\}+B_{i} N\left\{m_{e_{i+1}}\right\}, \\
\frac{\partial N\left\{m_{e_{i}}\right\}}{\partial t}= & A_{i} s\left(N\left\{m_{g_{i}}\right\}-N\left\{m_{e_{i}}\right\}\right)-A_{i} N\left\{m_{e_{i}}\right\} \\
& -C_{i+1} N\left\{m_{e_{i}}\right\}-B_{i-1} N\left\{m_{e_{i}}\right\}+N\left\{m_{e_{i-1}}\right\} \\
& +N\left\{m_{e_{i+1}}\right\}-2 N\left\{m_{e_{i}}\right\} .
\end{aligned}
$$

In (1), the magnetic sublevels $m_{F_{e}}= \pm 5$ remained unpopulated because of the linear polarization excitation $(\pi$-type of transitions) and because of the absence of population exchange between $m_{F_{e}}= \pm 5$ and the other sublevels. Note that for (2), the number $i$ takes additional values $i=$ $-5, \ldots,+5$ because atoms from the neighboring excited state sublevels populate the $m_{F_{e}}= \pm 5$ sublevels. The result of simulation is presented in Figure 9.

Figure 9(a) shows completely different distribution of the population density between Zeeman sublevels of the $F_{g}=$ 4 level when compared to the result obtained in absence of elastic interactions between Cs atoms (Figure 6(a)). It can be clearly seen that due to the mixing process, the atoms being accumulated on $m_{F_{g}}=0$-the sublevel with highest transition probability are now distributed to the other ground Zeeman sublevels. At the same time, the now populated $m_{F_{e}}= \pm 5$ levels relax only to the ground sublevels $m_{F_{g}}= \pm 4$ introducing an additional contribution for those particular levels. This contribution is the reason to have higher population density on $m_{F_{g}}= \pm 4$, compared to the neighboring sublevels, being almost equal to the $m_{F_{g}}=0$ population density.

Hence, the situation has changed in the following three aspects:

(i) due to the elastic interactions of Cs atoms, the population of the sublevel with the highest probability 


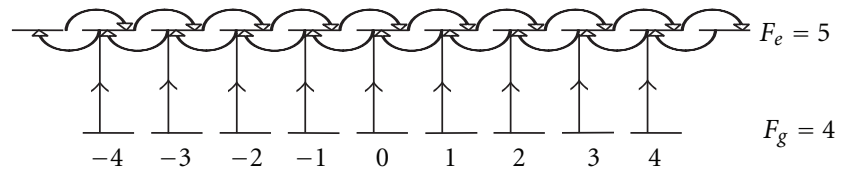

FIgURE 8: Illustration of the excited level magnetic sublevel population mixing involved by the elastic interactions between the Cs atoms and/or between Cs-atom and the cell windows when the atom is traveling parallel to the windows surface in the ETC.

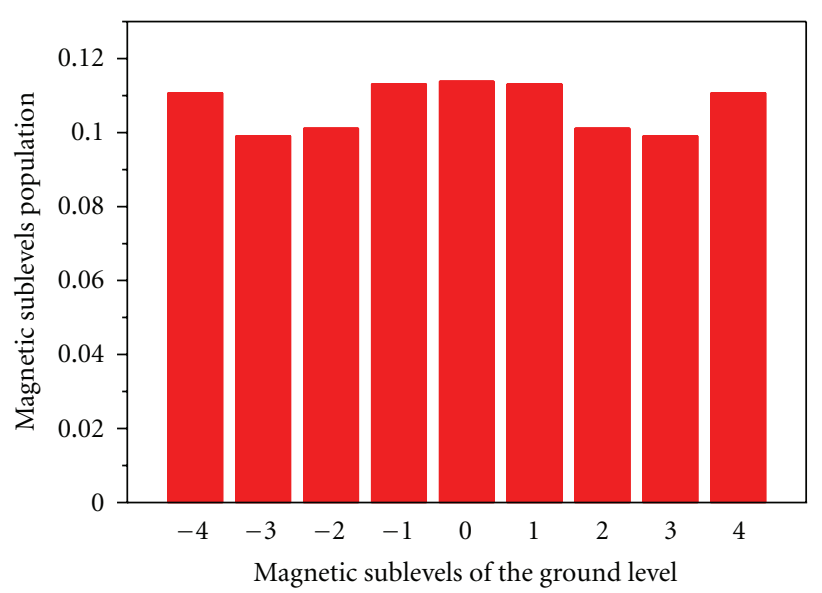

(a)

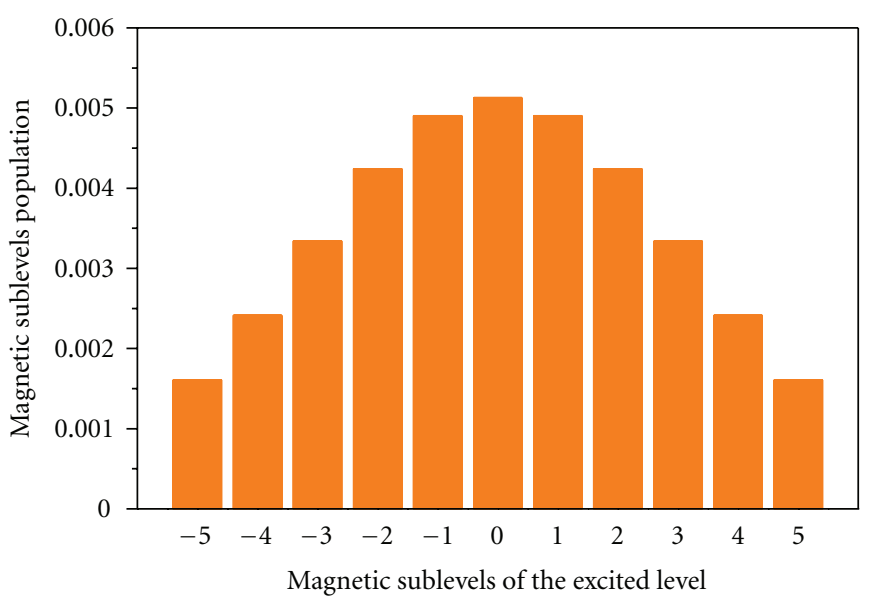

(b)

Figure 9: Illustration of the atomic population distribution between the Zeeman sublevels of the ground level $F_{g}=4$ (a) and of the $F_{e}=5$ excited level (b), for the $F_{g}=4 \rightarrow F_{e}=5$ transition. The collision rate leading to the population density redistribution used is of $10 \mathrm{MHz}$. Note the essential change in the atomic population distribution on the ground level magnetic sublevels compared to the case of the absence of elastic interactions (Figure 6).

of excitation $m_{F_{g}}=0$ is two times smaller than that shown in Figure 6(a);

(ii) the $m_{F_{g}}= \pm 4$ sublevels accumulate essential part of atomic population;

(iii) but the $m_{F_{g}}= \pm 4$ sublevels have the lowest excitation probability.

Based on the listed processes, we can conclude that the excitation efficiency will drop down, for the $B=0$ case. With imposed magnetic field scanned across $B=0$, the excited magnetic sublevel population mixing will lead to a reduction of the EIA resonance amplitude to its vanishing or even transformation to the EIT resonance. Presented model does not take into account the coherent processes nor the inelastic collisions of the atoms with the cell windows. It does not take into account, as well, the influence of the magnetic field that will additionally transfer the population density to the ground-state magnetic sublevels with higher $m_{F g}$. Including these factors in the theoretical model will stimulate the transformation of the EIA resonance to the EIT one.

\section{Influence of the Thin-Cell Walls}

In the theoretical model, we have not distinguished the two main processes that can be responsible for the excited level magnetic sublevel population mixing, namely, (i) the elastic interaction between Cs atoms at enhanced vapor pressures and (ii) the elastic interaction between the cell windows and the Cs atoms traveling parallel to the windows surface. In this section we would like to discuss in more details the influence of the thin-cell windows on the excited state population mixing. This role is particularly important and most probably dominating when lower atomic source temperatures are used in the experiment. Our calculations show that for $T=34^{\circ} \mathrm{C}$ (see Table 1), the number of elastic collisions between Cs atoms is very small because the time interval for such collisions to happen is comparable to the interaction time between the atom and the laser beam. However, the experimental results show that even for such a low temperature, the resonance sign reversal takes place.

Figure 10 compares the experimentally observed resonance sign reversal process in $L=700 \mu \mathrm{m}$ cell for two different temperatures $\left(34^{\circ} \mathrm{C}\right.$ and $\left.40^{\circ} \mathrm{C}\right)$, both not sufficient for introducing an essential number of Cs-Cs elastic collisions. It can be seen that for cell with thickness $L=700 \mu \mathrm{m}$ at very small laser intensities, the EIA resonance is observed like in the conventional cells. However, the sign of the resonance changes when increasing the laser intensity. This behavior leads us to the assumption that when the number of atoms on the excited state increases, it becomes possible to measure experimentally the result of physical processes involving the depolarization of the excited atoms. As a basic depolarization process in this case, we consider the atom-window elastic collisions. Further on, the experiment shows that at higher temperature (Figure 10(b)) the sign 


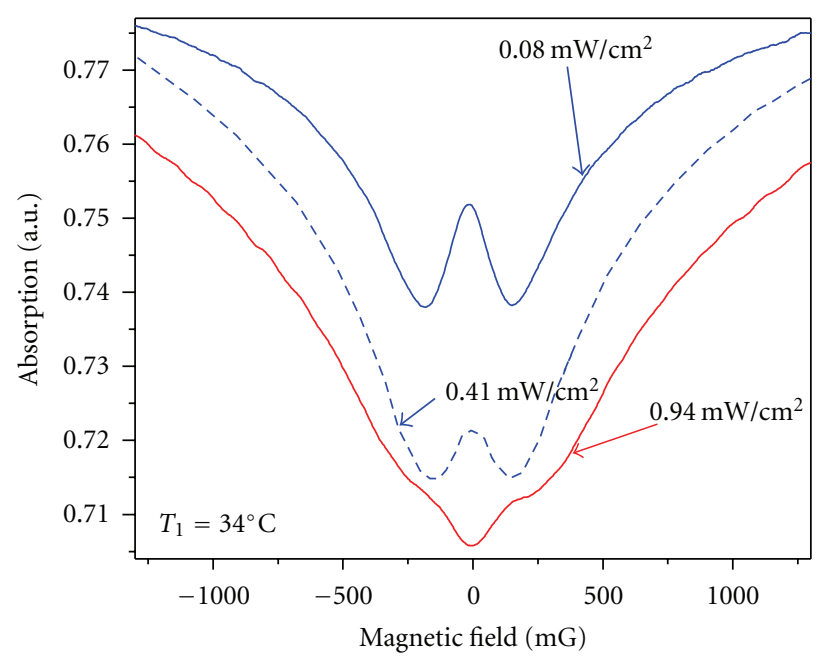

(a)

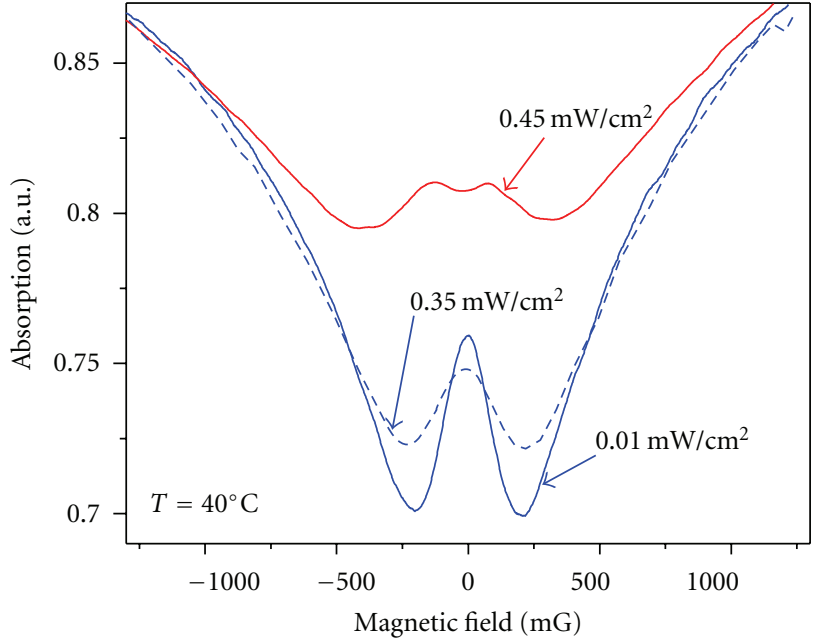

(b)

Figure 10: Sign reversal of the CPT resonance with the light intensity observed for the $F_{g}=4 \rightarrow F_{e}=5$ transition in $L=700 \mu \mathrm{m}$ cell. Two different temperatures of the atomic source are used: $34^{\circ} \mathrm{C}$ (a) and $40^{\circ} \mathrm{C} \mathrm{(b).} \mathrm{At} 40^{\circ} \mathrm{C}$, the sign-reversal process is observed for lower light intensity $\left(0.45 \mathrm{~mW} / \mathrm{cm}^{2}\right)$ as compared to $T=34^{\circ} \mathrm{C}$, where the effect is observed at $0.94 \mathrm{~mW} / \mathrm{cm}^{2}$.

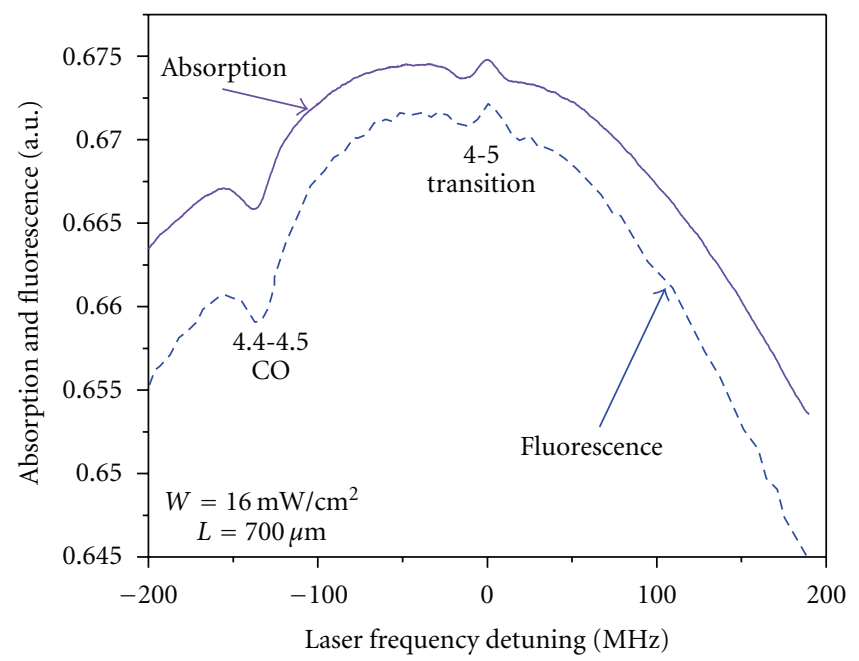

(a)

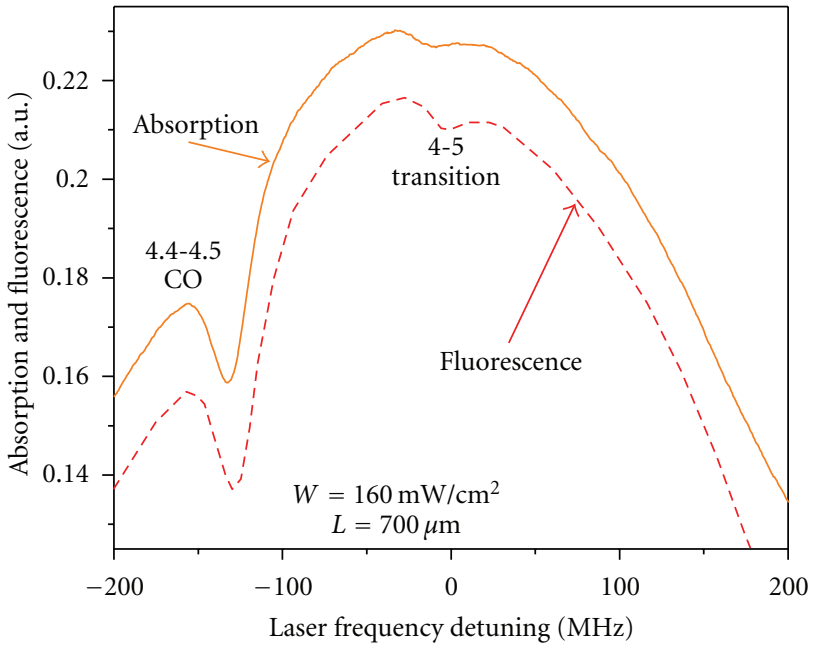

(b)

FIGURE 11: Sign reversal of the velocity selective optical pumping resonance with light intensity, observed at the $F_{g}=4 \rightarrow F_{e}=5$ transition in $L=700 \mu \mathrm{m}$ cell. Cell temperatures $T=25^{\circ} \mathrm{C}$. Laser light intensity: $W=16 \mathrm{~mW} / \mathrm{cm}^{2}$ (a) and $W=160 \mathrm{~mW} / \mathrm{cm}^{2}(\mathrm{~b}) . \mathrm{CO}$ denotes the cross-over resonance for both light intensities.

TABLE 1: Calculated values for ${ }^{133}$ Cs: vapor pressure $P$, atomic density $\rho$, most probable Cs atomic velocity $v$, collision rate $r_{\text {coll }}$, mean free path between the collision of two Cs atoms $\lambda$, time between two atomic collisions $\tau_{\text {int }}$ with the cell wall (having $d$ : the thin-cell window diameter), time between two atom collisions $\tau_{\text {coll }}$, and the number of collisions $n_{\text {collCs }}$ Cs-Cs during a complete flight along the cell diameter $d$.

\begin{tabular}{lcr}
\hline & $T_{1}=120^{\circ} \mathrm{C}=393 \mathrm{~K}$ & $T_{2}=34^{\circ} \mathrm{C}=307 \mathrm{~K}$ \\
\hline$P(\mathrm{~Pa})$ & $0.254695 \mathrm{~Pa}$ & $0.42 * 10^{-3} \mathrm{~Pa}$ \\
$\rho\left(\mathrm{cm}^{-3}\right)$ & $4.44 * 10^{13} \mathrm{~cm}^{-3}$ & $9.022 * 10^{10} \mathrm{~cm}^{-3}$ \\
$\bar{\nu}=\sqrt{2 k_{B} T / M}(\mathrm{~m} / \mathrm{s})$ & $221.7 \mathrm{~m} / \mathrm{s}$ & $196.01 \mathrm{~m} / \mathrm{s}$ \\
$r_{\text {coll }}=P(T) /\left(k_{B} T \sigma_{k} \bar{\nu}\right)(1 / \mathrm{s})$ & $9.84 * 10^{6} 1 / \mathrm{s}$ & $1.8 * 10^{4} 1 / \mathrm{s}$ \\
$\lambda=\bar{\nu} / r_{\text {coll }}(\mathrm{m})$ & $22.5 * 10^{-6} \mathrm{~m}$ & $9.8 * 10^{-3} \mathrm{~m}$ \\
$\tau_{\text {int }}=d / v(\mathrm{~s})$ & $4.5 * 10^{-5} \mathrm{~s}$ & $5 * 10^{-5} \mathrm{~s}$ \\
$\tau_{\text {coll }}=\lambda / v(\mathrm{~s})$ & $1 * 10^{-7} \mathrm{~s}$ & $5 * 10^{-5} \mathrm{~s}$ \\
$n_{\text {collCs }}=\tau_{\text {int }} / \tau_{\text {coll }}$ & 450 & 1 \\
\hline
\end{tabular}




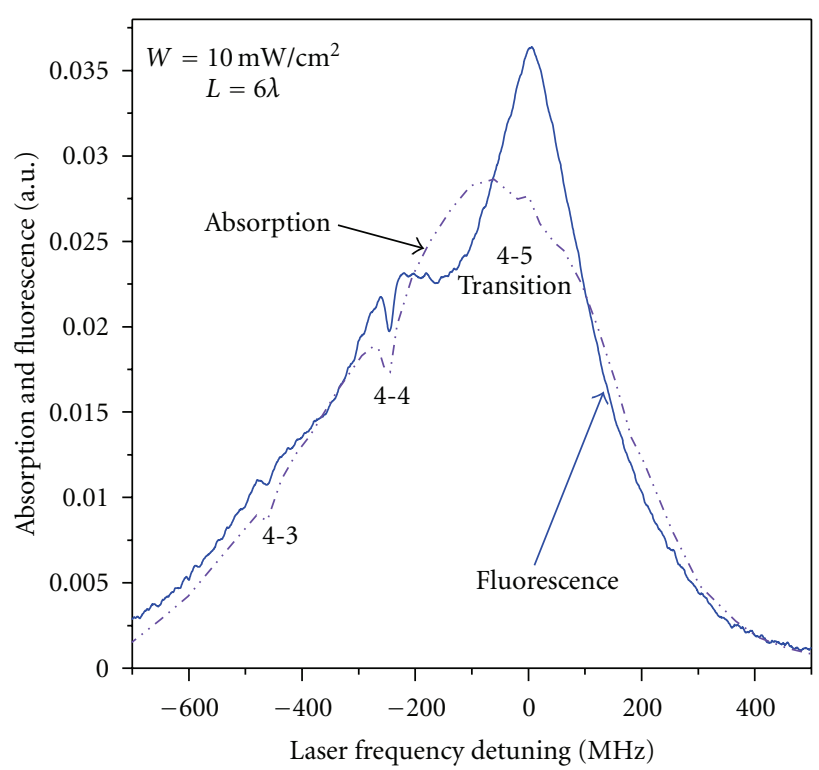

(a)

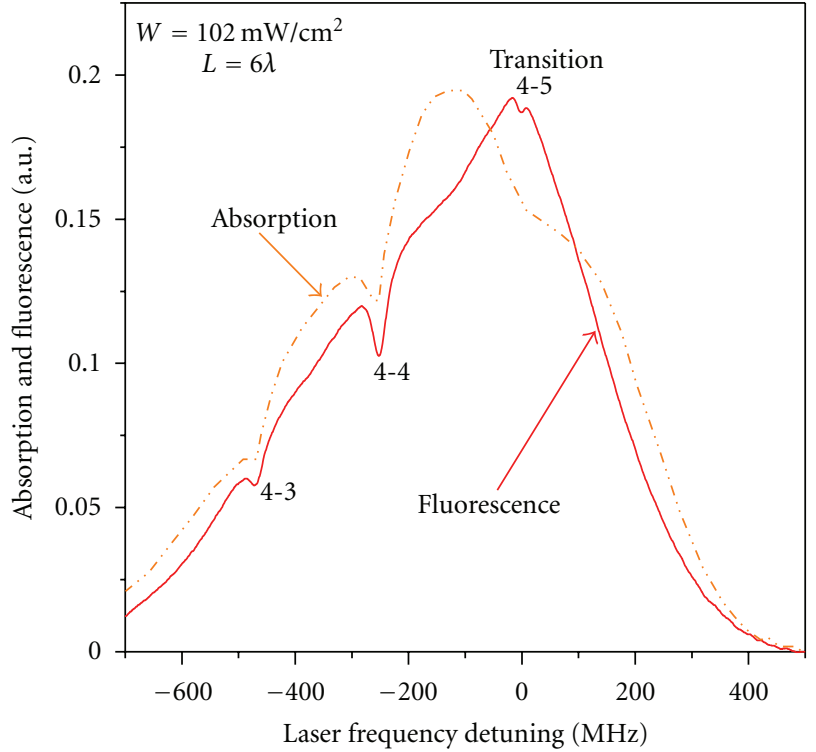

(b)

FIGURE 12: Sign reversal of the velocity selective optical pumping resonance with light intensity, observed for the $F_{g}=4 \rightarrow F_{e}=5$ transition, in $L=6 \lambda$ cell $(\lambda=852 \mathrm{~nm})$. Atomic source temperature $T=49^{\circ} \mathrm{C}$. Light intensity: $W=10 \mathrm{~mW} / \mathrm{cm}^{2}$ (a) and $W=102 \mathrm{~mW} / \mathrm{cm}^{2}(\mathrm{~b})$.

reversal happens at lower light intensity. The increase of the temperature leads to higher atomic concentration allowing higher population of the excited state. The latest contribute to an earlier switch from the EIA resonance to an EIT one.

When atoms are moving in the cell, they can undergo both elastic and inelastic collisions with the cell windows. In this way, the spectroscopy of thin cell provides the possibility to study the atom-surface interactions. If the atom flies very close to the surface, it can feel the periodic potential of the crystal surface of the thin cell. The effect which is known, up to now, concerning atom-surface interaction is the long-range van der Waals (vdW) attraction between the atom (in ground or excited state) and the dielectric surface (YAG windows) when the distance is $\sim 100 \mathrm{~nm}$. This effect may cause a shift or broadening of the atomic transition, both in transmission and in fluorescence [11]. So far, the influence of the long-range vdW force on the polarization of the excited state has not been investigated. In our work, we consider such influence on polarization as probable in analogy with the behavior of the bright CPT resonance in conventional buffered cell containing Cs atoms. It has been shown [2] that the bright CPT resonance observed in evacuated conventional cells transforms into a dark resonance when buffer gas is added. The resonance sign reversal there is attributed to depolarization of the excited state by the elastic collisions between alkali and noble gas atoms. It is well known that the last type of collisions causes also shift and broadening of atomic transitions.

In our model of elastic collisions, we make the following assumptions: (i) the elastic collisions do not affect the ground-state population and do not change the electron and nuclear spin; (ii) the elastic collisions redistribute only the population between the magnetic sublevels of the excited state. In Table 1 , it was shown that for $T_{2}=34^{\circ} \mathrm{C}$, the elastic collisions between Cs atoms can be neglected. Therefore, the observed excited state depolarization could be attributed to the elastic interaction between the Cs atoms and the thin-cell windows.

\section{Narrow Velocity Selective Optical Pumping Resonance Sign Reversal}

To put more light on the excited state depolarization processes in micrometric cells, here we present experimental measurement of the absorption and fluorescence spectra at the $F_{g}=4$ set of transitions. Figure 11 shows the absorption and fluorescence spectra obtained in $L=700 \mu \mathrm{m}$ cell, for the $F_{g}=4 \rightarrow F_{e}=5$ transition, at low atomic source temperature $T=25^{\circ} \mathrm{C}$. At such a temperature, the only factor for the excited state depolarization remains the elastic interaction between atoms and the cell windows. For low light intensity, a very well-pronounced narrow pick in the fluorescence, as well as in the absorption, takes place (Figure 11(a)). The presence of this bright structure shows that the excited state depolarization does not take place under these conditions. This very interesting narrow pick is rarely observed [12, 13]. It is due to the fact that the $F_{g}=4 \rightarrow F_{e}=5$ transition is completely closed. In such a case, the excited atoms can just turn back to their initial ground level by the spontaneous emission. However, during the spontaneous decay, a velocity selective accumulation of "slow" atoms to the ground magnetic sublevels takes place, leading to the formation of the narrow pick. Further on, we call these narrow enhanced absorption and fluorescence features: bright velocity selective optical pumping (VSOP) resonances. Note that in Figure 11(a), the bright VSOP resonance is accompanied by a VSOP resonance of opposite sign. The reduced absorption/fluorescence VSOP resonance is related 
to cross-over $(\mathrm{CO})$ resonance occurring between $F_{g}=$ $4 \rightarrow F_{e}=4$ and $F_{g}=4 \rightarrow F_{e}=5$ transitions. Due to the small thickness of the cell, there is a good overlapping of the pump beam and the beam reflected by the second window. This causes appearance of $\mathrm{CO}$ resonances in the spectrum that is somehow unexpected at first glance for a single beam experiment.

It is very interesting to note that the increase of the laser intensity with one order of magnitude $\left(160 \mathrm{~mW} / \mathrm{cm}^{2}\right)$ leads to transformation of the former bright VSOP resonance to a dark VSOP one, that is, reduced absorption or fluorescence resonance. The dark VSOP resonances are much broader than the previously observed bright VSOP structure at low power. Therefore, in this experiment, it is difficult to distinguish between the two processes: the saturation of optical transition and the excited state depolarization.

The absorption and fluorescence behavior as a function of laser intensity was studied also for essentially thinner cell with $L=6 \lambda(\lambda=852 \mathrm{~nm})$. This is the lowest sector of the multipurpose cell (Figure 2 right). The goal of this experiment is to enhance the portion of atoms influenced by the cell windows and, at the same time, to work at the reduced cell temperatures, which is not possible when using the $L=\lambda$ cell. Figure 12 shows the absorption and fluorescence spectra obtained in the $L=6 \lambda$ cell, for the $F_{g}$ $=4$ set of transition, at $T=49^{\circ} \mathrm{C}$. For low light intensity and the two open transitions $\left(F_{g}=4 \rightarrow F_{e}=3,4\right)$, the formation of dark VSOP resonances is clearly seen. At the same time, the $F_{g}=4 \rightarrow F_{e}=5$ transition exhibits wellpronounced bright VSOP resonances in the absorption and the fluorescence centered at the optical transitions.

It is again evident that when increasing the laser intensity, the bright VSOP resonances in the laser absorption and fluorescence transform to dark VSOP features, for the $F_{g}=$ $4 \rightarrow F_{e}=5$ transition. However, in this cell, the dark VSOP resonance in the fluorescence is much narrower than that observed in the absorption. We relate this result to essentially higher anisotropy expressed in $L=6 \lambda$ cell compared to the $L=700 \mu \mathrm{m}$ cell. It is worth to stress that at higher intensity $\left(102 \mathrm{~mW} / \mathrm{cm}^{2}\right)$, the dark VSOP resonance in the fluorescence is not broader at all than the bright VSOP resonance in the fluorescence, observed at low light intensity $\left(10 \mathrm{~mW} / \mathrm{cm}^{2}\right)$. This experimental result is considered in support of the depolarizing factor of the excited state. Therefore, we can assume that when the population density of the excited state is increased essentially, better conditions for depolarization of the slow atoms are provided, that can be experimentally observed. Such a depolarization does not involve a considerable VSOP resonance broadening. Further study still in progress aimed at experimental confirmation of the depolarization of the excited state (of the $F_{g}=4 \rightarrow F_{e}=$ 5 transition) caused by the influence of the cell windows.

\section{Conclusions}

The miniaturization of practical devices based on alkali atomic vapor confined in optical cells is of rising interest for the development of photonic sensors. In this paper, we discuss how the optical cell thickness miniaturization process reflects on the parameters of the coherent resonances observed in Cs-filled vacuum cells.

Compared to Cs spectra obtained by means of conventional cells, essential and even principal differences can occur in case of micrometric cells. We have shown that the cell with thickness of $L=700 \mu \mathrm{m}$ takes an intermediate place between the conventional cell and the extremely thin cell with thickness of the order of the optical wavelength $(L \sim \lambda)$. The EIA resonances are observed on the $F_{g}=4 \rightarrow F_{e}=$ 5 transition in the most widely used conventional cells. However, the EIT resonance has been reported for the $L=\lambda$ cell. Here, we demonstrate (for $L=700 \mu \mathrm{m}$ cell) how the coherent resonance obtained at the same optical transition presents the particular behavior of sign reversal when the intensity of the laser radiation increases. This peculiarity is obtained for reduced cell temperatures equal or slightly above the room temperature. The physical processes and the possible reasons behind this effect of the resonance sign reversal are discussed and analyzed.

A theoretical model is developed, taking into account the mixing of the population density of the excited state magnetic sublevels. For low atomic concentration, this mixing process is attributed to the elastic interactions between Cs atoms and the cell windows. At elevated temperature of the atomic source, the probability for contribution from the CsCs elastic interaction strongly increases.

Additional factor influencing the coherent resonance formation is the laser intensity. It is shown experimentally that the laser intensity increasing (i.e., more atoms in the excited state) results in the EIA resonance transformation to EIT one. We suggest that the redistribution of the ground magnetic sublevel population is stimulated by the enhanced number of depolarized atoms on the excited state. As a final result of all those processes, the atoms being accumulated to the ground magnetic sublevels with higher excitation probability at low light intensity are redistributed to sublevels with low excitation probability with the intensity rising. In this way, the increased number of absorbed atoms can be experimentally observed as resonance sign reversal.

As a confirmation of the sign-reversal process based on the depolarization of the excited state, the analysis is presented of the fluorescence and absorption spectra, in case of micrometric cells with two different levels of miniaturization ( $L=700 \mu \mathrm{m}$ and $L=5 \mu \mathrm{m})$.

The experimental results and the analysis performed show a potential for studying the atom-surface interaction using the coherent resonance parameters and their behavior.

\section{Acknowledgments}

The authors would like to express their gratitude to professor David Sarkisyan (National Academy of Sciences of Armenia) for building and offering to them the unique micrometric and nanometric cells. The work is partially supported by the Bulgarian NCSR (Grant no. DO 02-108/22.05.2009) and Indian-Bulgarian (BIn-2/07) bilateral contract. 


\section{References}

[1] A. Papoyan, M. Auzinsh, and K. Bergmann, "Nonlinear Hanle effect in Cs vapor under strong laser excitation," European Physical Journal D, vol. 21, no. 1, pp. 63-71, 2002.

[2] C. Andreeva, S. Cartaleva, Y. Dancheva et al., "Coherent spectroscopy of degenerate two-level systems in Cs," Physical Review A, vol. 66, no. 1, Article ID 012502, pp. 1250211250212, 2002.

[3] D. Sarkisyan, D. Bloch, A. Papoyan, and M. Ducloy, "SubDoppler spectroscopy by submicron thin Cs-vapor layer," Optics Communications, vol. 200, pp. 201-208, 2001.

[4] C. Andreeva, S. Cartaleva, L. Petrov et al., "Saturation effects in the sub-Doppler spectroscopy of cesium vapor confined in an extremely thin cell," Physical Review A, vol. 76, no. 1, article 013837, 2007.

[5] C. Andreeva, A. Atvars, M. Auzinsh et al., "Ground-state magneto-optical resonances in cesium vapor confined in an extremely thin cell," Physical Review A, vol. 76, no. 6, Article ID 063804, 2007.

[6] L. Lenci, A. Lezama, and H. Failache, "Dark resonances in thin cells for miniaturized atomic-frequency references," Optics Letters, vol. 34, no. 4, pp. 425-427, 2009.

[7] A. Sargsyan, M. G. Bason, D. Sarkisyan, A. K. Mohapatra, and C. S. Adams, "Electromagnetically induced transparency and two-photon absorption in the ladder system in thin columns of atomic vapors," Optics and Spectroscopy, vol. 109, no. 4, pp. 529-537, 2010.

[8] F. Renzoni, C. Zimmermann, P. Verkerk, and E. Arimondo, "Enhanced absorption Hanle effect on the $\mathrm{F}_{g}=\mathrm{F} \rightarrow \mathrm{F}_{e}=$ F + 1 closed transitions," Journal of Optics B: Quantum and Semiclassical Optics, vol. 3, no. 1, pp. S7-S14, 2001.

[9] Daniel A. Steck, "Cesium D Line Data," (23 January 1998 , revision 1.6, 14 October 2003), http://steck.us/alkalidata /cesiumnumbers.1.6.pdf.

[10] D. G. Slavov, S. S. Cartaleva, D. Sarkisyan, P. N. Ghosh, B. Ray, and S. Mitra, "Rubidium vapor cell miniaturization-influence on the optical pumping process," Indian Journal of Physics, vol. 84, no. 8, pp. 1083-1093, 2010.

[11] I. Hamdi, P. Todorov, A. Yarovitski et al., "Laser spectroscopy with nanometric gas cells: distance dependence of atomsurface interaction and collisions under confinement," Laser Physics, vol. 15, no. 7, pp. 987-996, 2005.

[12] S. Cartaleva, S. Saltiel, A. Sargsyan et al., "Sub-Doppler spectroscopy of cesium vapor layers with nanometric and micrometric thickness," Journal of the Optical Society of America B, vol. 26, no. 11, pp. 1999-2006, 2009.

[13] V. Biancalana, S. Cartaleva, Y. Dancheva et al., "Population loss in closed optical transitions of $\mathrm{Rb}$ and $\mathrm{Cs}$ atoms confined in micrometric thin cells," Acta Physica Polonica A, vol. 116, no. 4, pp. 495-497, 2009. 

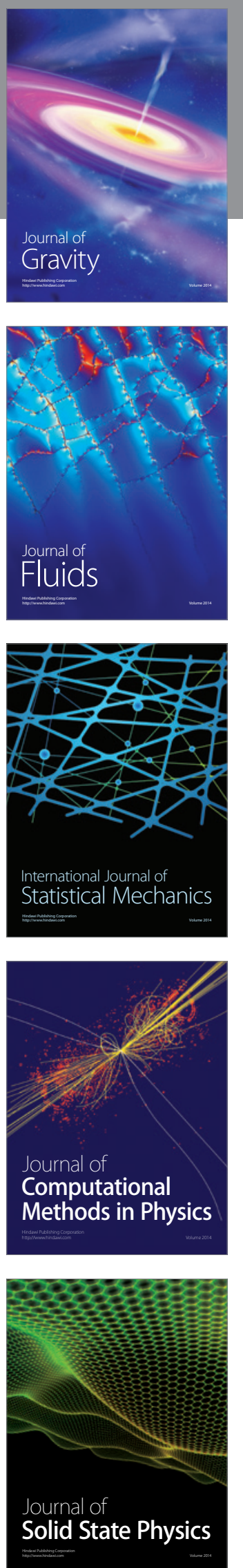

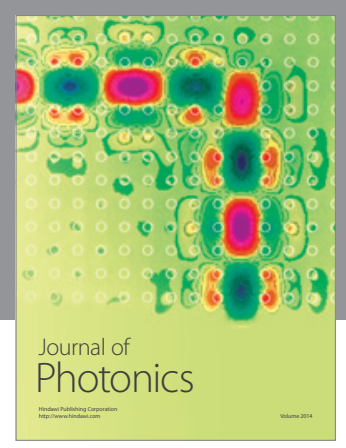

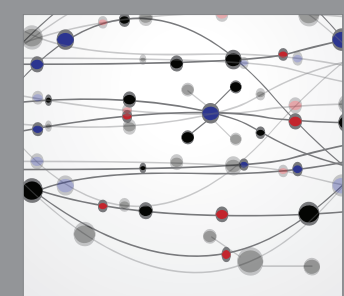

The Scientific World Journal
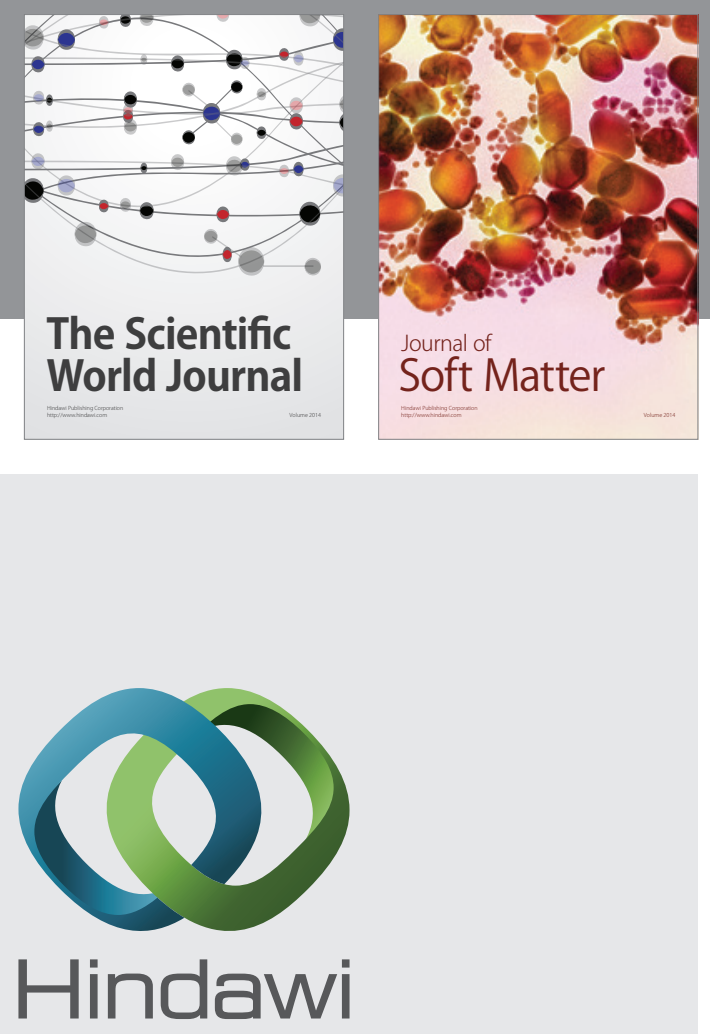

Submit your manuscripts at

http://www.hindawi.com
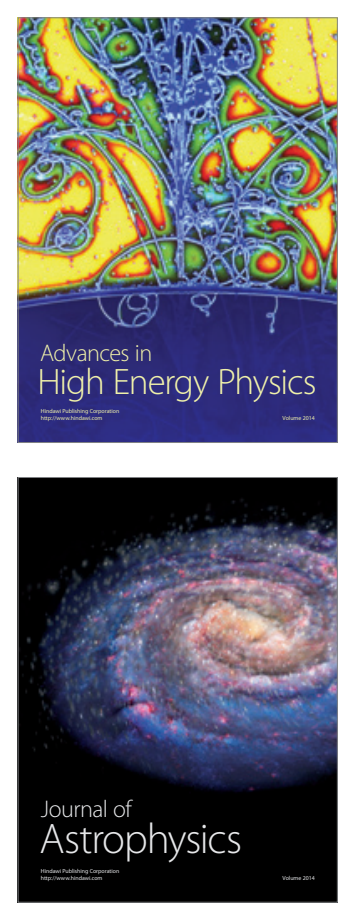
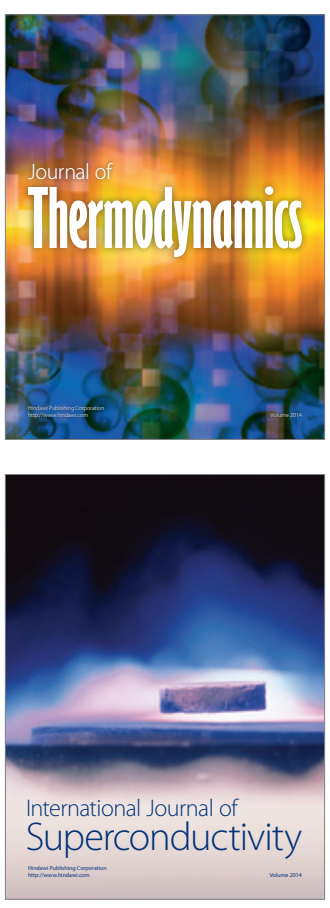
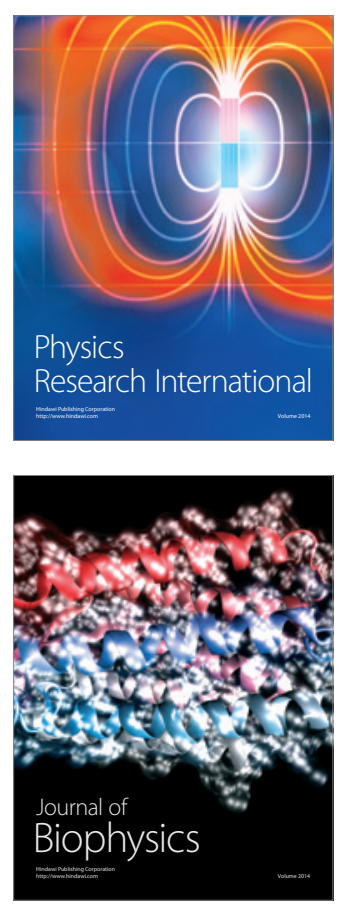
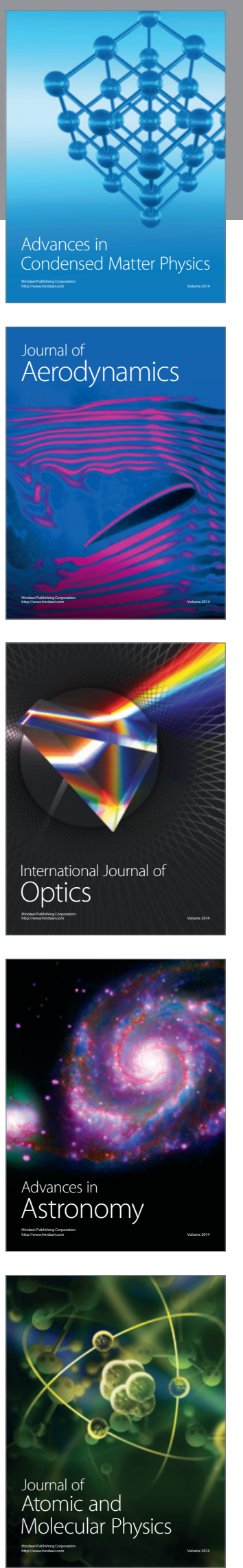